\title{
Direct resin composite restorations for fractured maxillary teeth and diastema closure: A 7 years retrospective evaluation of survival and influencing factors
}

\author{
Edina Lempel ${ }^{a, *}$, Bálint Viktor Louász ${ }^{a}$, Réka Meszarics ${ }^{a}$, Sára Jeges ${ }^{c}$, \\ Ákos Tóth ${ }^{c}$, József Szalma ${ }^{b}$ \\ a Department of Restorative Dentistry and Periodontology, University of Pécs, Pécs, Hungary \\ ${ }^{b}$ Department of Oral and Maxillofacial Surgery, University of Pécs, Pécs, Hungary \\ c Faculty of Sciences, University of Pécs, Pécs, Hungary
}

\section{A R T I C L E I N F O}

\section{Article history:}

Received 21 November 2016

Received in revised form

12 February 2017

Accepted 13 February 2017

Available online xxx

\section{Keywords:}

Fracture

Diastema

Resin based composite

USPHS criteria

Longevity

\begin{abstract}
A B S T R A C T
Objectives. This retrospective study evaluated the survival rate of anterior direct resin based composite (RBC) build-ups in vital teeth made of microhybrid and nanofill RBC materials and the influence of bruxism, beverage consumption and smoking on the long-term performance of restorations.

Methods. Patients receiving anterior restoration between 2006 and 2011, with the diagnosis of fracture or diastema, were selected. A total of 65 adult patients (mean age: 25.2) with 163 restorations (78 Filtek Supreme XT and 85 Enamel Plus HFO) were evaluated using the USPHS criteria. Data were analyzed with Fisher's Exact Test, Extended Cox-regression analysis and Kaplan-Meier method.

Results. Mean observation period was $7.2( \pm 1.4)$ years and the mean annual failure rate for this period was $1.43 \%$. The reasons of failures included restoration fracture and color mismatch. Nanofill restorations had significantly higher rate of color mismatch $(p=0.002)$, microhybrids more frequently failed in fracture of restoration $(p=0.034)$. The overall difference in potential hazard of using Enamel Plus HFO or Filtek Supreme XT was not significant $(p=0.704)$. Chipping or fracture of the restoration was more frequent in the first year after placement $(p=0.036)$, while beverage consumption was significantly correlated with discoloration of the restorations $(p=0.005)$.

Significance. The application of direct RBC restorations provides an excellent treatment option for fractured teeth and for closing diastemas. The overall survival rate was $88.34 \%$ up to 10 years. Microhybrid and nanofill RBC restorations showed similar survival rates, however nanofills discolored at a higher rate, meanwhile chipping of the restoration occurred frequently with microhybrids.
\end{abstract}

๔ 2017 The Academy of Dental Materials. Published by Elsevier Ltd. All rights reserved.

\footnotetext{
* Corresponding author at: Department of Restorative Dentistry and Periodontology, University of Pécs, 5. Dischka Gy Street, Pécs H-762, Hungary. Fax: +36 72535905 .

E-mail address: lempel.edina@pte.hu (E. Lempel).

http://dx.doi.org/10.1016/j.dental.2017.02.001

0109-5641/@ 2017 The Academy of Dental Materials. Published by Elsevier Ltd. All rights reserved.
} 


\section{Introduction}

Patients suffering from an anterior fracture, attrition, diastema or dental malformation and malposition should be provided with adequate esthetic correction including orthodontic treatment, indirect ceramic or direct resin based composite (RBC) restorations. Recently, with the continuous development of adhesives and RBC technology the state-ofthe-art treatment option in operative dentistry for the esthetic improvements of healthy teeth - especially for adolescents and young adults with intact enamel - can be non-invasive or at least minimally invasive [1,2]. Beside the excellent esthetic and mechanical features of the different types of RBCs, the dentist's skill in achieving a natural anatomical shape, surface texture and shade is also a prerequisite for an esthetically pleasing result. Compared to ceramic restorations the direct applications with RBCs have several benefits, such as quickness, cheapness and easy of repair. Currently, RBC is the first choice material to restore anterior and posterior teeth $[2,3]$.

Clinical data on the performance of posterior RBC restorations are indicating low annual failure rates (AFRs) and long-lasting survival [4]. In contrast, despite the general application of RBCs in the anterior region, there is a lack of evidence from clinical trials especially regarding the long term performance of non-carious anterior restorations. These direct tooth-shaped restorations seem to be used increasingly in clinical practice with excellent short-term results [5]. However, a demand for knowledge still exists regarding the potential influencing factors for failure in the long-term. The main reported reasons for failure in posterior teeth are secondary caries and fracture with 70-98\% survival rate after 8 and 22 years $[6,7]$. However, in contrast, caries is not a major cause for failure of anterior restorations [8]. In studies looking at build-ups or direct veneers esthetic failures were more frequently observed, where color alterations, surface staining, and marginal discoloration could negatively influence the patient's perception of the restoration $[9,10]$. On the other hand, Wolff et al. and van Dijken et al. found that the most frequent threat to direct composite build-ups is the fracture of the RBC $[5,11]$. In case of chipping, due to the composite's material properties, a simple repair can be performed to extend the life of the original restoration. These unfavorable events could be classified in the evaluation process as survival. The 3-5 years survival of anterior restorations could vary between $79-89 \%[5,8,12]$. However, the potential influence of formulation characteristics of RBC, the size of the build-ups, the patient's factors and operator characteristics remain to be determined, especially in long-term clinical trials. Kubo et al. investigated only the factors associated with the longevity of Class III, IV and V RBC restorations with respect to the gender, age, operator factor, cavity type and retreatment risk [13]. They concluded that operator factor, cavity type and retreatment risk had significant influence on the survival time. Focusing on the material, Gresnigt et al. compared two microhybrid RBC materials in their short-term study and did not find differences in the longevity [9].

There are several research techniques for the assessment of restoration longevity. Among others these include retrospective, prospective studies, randomized controlled clinical trials, cohort studies and cross-sectional analysis. The biggest challenge for long-term studies is the wear out of the study populations. Retrospective longitudinal studies in particular allow us observation times of more than 10 years, while also enabling us to examine many restorations in a relatively short time $[3,6,14,15]$. However, retrospective studies do seem to be inferior to prospective ones in certain aspects. In the former design there is an obvious lack of standardization of indication and treatment protocols. Although, if the conditions are set out well at the start, and the number of examining operators are kept to a minimum, the potential of a certain type of restoration can still be reflected [3].

The purpose of this retrospective study was to investigate the failures and estimate the survival of direct RBCs placed for the restoration of fractured maxillary anterior teeth or placed for closing diastemas according to the modified USPHS criteria, in clinical practice using a nanofill RBC and a microhybrid RBC. Factors thought to be associated with failure such as the size of the build-up, bruxism, dietary habits and smoking were also examined for up to ten years.

\section{Material and methods}

\subsection{Study design and participants}

The database with clinical records from the Operative Dentistry Department at the University of Pécs was used in the present evaluation. From this database, all patients who had received direct $\mathrm{RBC}$ restoration in the maxillary anterior teeth by the first author (E.L.) for fracture or diastema closure (including peg-shaped lateral incisors) were selected for this retrospective analysis. The study protocol was approved by the Regional Research Ethics Committee of University of Pécs (3410.1./2009). All patients were contacted by phone or mail. Those patients who were able to participate in the study, signed a written, informed consent prior to the start of the clinical evaluation.

\subsection{Inclusion and exclusion criteria}

For this retrospective study, a total of 65 patients with ages ranging from 18 and 58 years old (25 males and 40 females, mean age: 25.2 at the time of restoration placement) were selected according to pre-determined inclusion criteria from the registers of a Hungarian clinical practice (University of Pécs), from June 2006 to December 2011, securing a minimum observation period of 5 years and the longest one of 10 years. The selected patients received a total of 163 direct RBC build-ups in their vital maxillary teeth. 70 central incisors and 22 lateral incisors were restored with the indication of fracture. Diastema closure was performed in 32 cases in central incisors, 31 cases in lateral incisors (including peg-shaped lateral incisors, $\mathrm{n}=5$ ) and 8 cases in canine. Information was given to each participants regarding the alternative treatment options. The inclusion criteria employed comprised of the following: all participants were at least 18 years old, able to read and sign the informed consent document, physically and psychologically able to tolerate the procedure. Furthermore, patients who were selected for the 


\begin{tabular}{|c|c|c|c|}
\hline Brand & Type & Manufacturer & Chemical composition \\
\hline Filtek Supreme XT & Nanofill composite & 3M ESPE, St Paul, MN, USA & $\begin{array}{l}\text { BisGMA, UDMA, TEGDMA, PEGDMA, } 72.5 \\
\text { w\% ( } 55.6 \mathrm{v} \% \text { ) } \\
\text { non-agglomerated/non-aggregated } 20 \mathrm{~nm} \\
\text { silica and } 4-11 \mathrm{~nm} \text { zirconia filler, } \\
0.6-20 \mu \mathrm{m} \text { aggregated cluster fillers }\end{array}$ \\
\hline Enamel Plus HFO & Micro-hybrid composite & Micerium S.p.A., Avegno, Italy & $\begin{array}{l}\text { BisGMA, TEGDMA, UDMA, } \\
\text { 1,4-butandiol-dimethacrylate, } 75 \mathrm{w} \% \text { ( } 53 \\
\text { v\%) } 0.7 \mu \mathrm{m} \text { glass filler and highly } \\
\text { dispersed } 0.04 \mu \mathrm{m} \text { silicone dioxide }\end{array}$ \\
\hline Adper Single Bond & Total-etch adhesive & 3M ESPE, St Paul, MN, USA & $\begin{array}{l}\text { BisGMA, UDMA, HEMA, glycerol } \\
\text { 1,3-dimethacrylate, methacrylate } \\
\text { functional copolymer of polyacrylic and } \\
\text { polyitaconic acids, } 10 \% 5 \mathrm{~nm} \text { silane } \\
\text { treated colloidal silica }\end{array}$ \\
\hline Ultra-Etch & Phosphoric acid & Ultradent Products Inc, South Jordan, UT, USA & $38 \%$ phosphoric acid \\
\hline
\end{tabular}

Abbreviations; BisGMA: bisphenol A diglycidil ether dimethacrylate; UDMA: diurethane dimethacrylate; TEGDMA: triethylene glycol dimethacrylate; PEGDMA: polyethylene glycol dimethacrylate.

study had full dentition and normal occlusion without generalized periodontal disease, as verified by the clinical and radiographic records, and these patients had remained in continuous clinical follow-up, including at least 1 annual recall without attending other dentists. Reasons for placement of direct RBC build-ups were either fracture of the tooth or diastema, including peg-shaped lateral malformation correction as well or changing old restorations with the same dental history. Margins placed on enamel was a requirement, had to be fulfilled in order for the placement of direct RBC. Endodontically treated teeth at baseline were excluded from the study, however the necessity of endodontic treatment after build-up was recorded from the documentation during the evaluation. Dental history of the restorable tooth was recorded from the clinical documentation. The extension of the $\mathrm{RBC}$ restoration was grouped as following: $<25 \%, 25-50 \%$ or $>50 \%$ of the entire anatomical crown.

\subsection{Restorative procedures}

The brands, types, manufacturers, chemical compositions of the materials used in this study are listed in Table 1.

All RBC restorations were performed by the first author (E.L.) specializing in restorative dentistry. Operative procedures were performed under local anesthesia if it was necessary. Few of the restorations were placed free-hand using Mylar strip, but most anterior build-ups were placed with the aid of a silicon stent constructed from a diagnostic wax-up. All RBCs had been placed following the principle of minimally invasive dentistry. Before tooth preparation the teeth were cleaned with pumice and the shade selection was performed with Vitapan Classical Shade Guide (Vita Zahnfabrik, Bad Säckingen, Germany) according to the corresponding shade selector for Filtek Supreme XT and chromatic chart for Enamel Plus HFO [16]. The bonding surfaces of the teeth were roughened with abrasive discs (Sof-Lex Contouring and Polishing Discs, 3M ESPE, St. Paul, MN, USA). In case of fracture, the unsupported enamel was removed with a red diamond needle-shaped bur (Dentsply Maillefer, Ballaigues,
Switzerland) and a long bevel (includes all enamel and up to half of the exposed dentin) was prepared on the buccal surface under constant water cooling. All margins were placed supragingivally to maintain good periodontal health. The teeth were isolated mostly with conventional rubber dam technique or with split dam technique or in some cases with lip retractor and cotton rolls. Regarding the adhesive technique, a twostep etch-and-rinse system was used for each restoration. The teeth were conditioned with total etch technique by applying $38 \%$ phosphoric acid (Ultra-etch, Ultradent, South Jordan, UT, USA). The acid gel was first applied on the enamel for $10 \mathrm{~s}$, followed by $10 \mathrm{~s}$ on both dentin and enamel. After $20 \mathrm{~s}$ rinsing and careful drying of the cavity with air was performed (wet bonding technique), one step enamel-dentin adhesive system (Adper Single Bond, 3M ESPE) was applied as per manufacturer's instructions by rubbing the dentin and enamel with a micro-brush soaked in the resin. In order to evaporate the solvent, gentle, $10 \mathrm{~s}$ air-drying was carried out, followed by polymerization with a light emitting diode (LED) curing unit ( $\lambda=420-480 \mathrm{~nm}$; LED.C, Woodpecker, Guilin, China) with $20 \mathrm{~s}$ exposure time at a light intensity of $1100 \mathrm{~mW} \mathrm{~cm}^{-2}$ and with an irradiated diameter of $10 \mathrm{~mm}$.

One microhybrid (Enamel Plus HFO, Micerium S.p.A., Avegno, Italy) and one nanofill (Filtek Supreme XT, 3M ESPE) $\mathrm{RBC}$ were used randomly, placed with layering technique. The restorations were gradually built up with a multilayer technique of dentin and enamel shades, additional "effect" shades with different opacity and translucency were applied when it was necessary for the natural appearance. Each enamel shade layer was light-cured for $20 \mathrm{~s}$, dentin shade layer for $40 \mathrm{~s}$ using the LED unit. The occlusion was checked in protrusive movements of the mandible. The final polishing was performed with fine-grit diamond burs to remove gross excess, followed by polishing with abrasive discs (Sof-Lex Finishing strips, 3M ESPE) and with aluminum oxide strips (Sof-Lex Finishing strips, 3M ESPE) for the interproximal surfaces. Finally, polishing brushes (Shiny S, Micerium S.p.A., Avegno, Italy) were used for the natural gloss until all restorations were considered clinically acceptable. 
Table 2 - List of modified United States Health Service (USPHS) criteria used for the clinical evaluations of the restorations.

\begin{tabular}{|c|c|c|c|}
\hline \multirow[t]{2}{*}{ Category } & \multicolumn{2}{|c|}{ Score } & \multirow[t]{2}{*}{ Criteria } \\
\hline & Acceptable & Unacceptable & \\
\hline Marginal adaptation & $\begin{array}{l}0 \\
1\end{array}$ & $\begin{array}{l}2 \\
3 \\
4\end{array}$ & $\begin{array}{l}\text { Smooth margin } \\
\text { All margins closed or posses minor voids, defects (enamel exposed) } \\
\text { Obvious crevice at margin, dentin or base exposed } \\
\text { Debonded from one end } \\
\text { Debonded from both ends }\end{array}$ \\
\hline Color match & $\begin{array}{l}0 \\
1\end{array}$ & $\begin{array}{l}2 \\
3 \\
4\end{array}$ & $\begin{array}{l}\text { Very good color match } \\
\text { Good color match } \\
\text { Slight mismatch in color or shade } \\
\text { Obvious mismatch, outside the normal range } \\
\text { Gross mismatch }\end{array}$ \\
\hline $\begin{array}{l}\text { Marginal } \\
\text { discoloration }\end{array}$ & $\begin{array}{l}0 \\
1\end{array}$ & $\begin{array}{l}2 \\
3\end{array}$ & $\begin{array}{l}\text { No discoloration evident } \\
\text { Slight staining, can be polished away } \\
\text { Obvious staining, cannot be polished away } \\
\text { Gross staining }\end{array}$ \\
\hline Surface roughness & $\begin{array}{l}0 \\
1\end{array}$ & $\begin{array}{l}2 \\
3\end{array}$ & $\begin{array}{l}\text { Smooth surface } \\
\text { Slightly rough or pitted } \\
\text { Rough, cannot be refinished } \\
\text { Surface deeply pitted, irregular grooves }\end{array}$ \\
\hline $\begin{array}{l}\text { Fracture of } \\
\text { restoration }\end{array}$ & 0 & $\begin{array}{l}1 \\
2 \\
3\end{array}$ & $\begin{array}{l}\text { No fracture } \\
\text { Minor crack lines or tiny chipping ( }<1 / 4 \text { of restoration) } \\
\text { Partial fracture of restoration ( }>1 / 4 \text { of restoration) } \\
\text { Debonding of restoration }\end{array}$ \\
\hline Fracture of tooth & $\begin{array}{l}0 \\
1\end{array}$ & $\begin{array}{l}2 \\
3\end{array}$ & $\begin{array}{l}\text { No fracture of tooth } \\
\text { Minor crack lines in tooth } \\
\text { Partial fracture of tooth ( }>1 / 4 \text { of crown) } \\
\text { Crown-root fracture (extraction) }\end{array}$ \\
\hline Wear of restoration & 0 & 1 & $\begin{array}{l}\text { No wear } \\
\text { Wear of restoration }\end{array}$ \\
\hline Wear of antagonist & 0 & 1 & $\begin{array}{l}\text { No wear } \\
\text { Wear of antagonist }\end{array}$ \\
\hline Caries & 0 & 1 & $\begin{array}{l}\text { No evidence of caries along the margin of the restoration } \\
\text { Caries evident continuous with the margin of the restoration }\end{array}$ \\
\hline $\begin{array}{l}\text { Post-operative } \\
\text { sensitivity }\end{array}$ & $\begin{array}{l}0 \\
1\end{array}$ & $\begin{array}{l}2 \\
3\end{array}$ & $\begin{array}{l}\text { No symptoms } \\
\text { Slight sensitivity } \\
\text { Moderate sensitivity } \\
\text { Severe pain }\end{array}$ \\
\hline
\end{tabular}

\subsection{Evaluation and statistical analysis}

The restorations were evaluated between June and September 2016 by two calibrated examiners using dental mirror and explorer, in accordance with modified United States Public Health Service (USPHS) criteria (Table 2) [9]. The dentists were trained and calibrated before the start of the evaluation. Cohen's kappa statistic was used to calculate observer agreement. Intraobserver (kappa values of 0.77 and 0.79 ) and interobserver's (a kappa value of 0.82) agreement was found excellent in this study. The history of the restorations was investigated from the dental records. If a restoration had failed, resulting in either replacement or repair, it was considered as failure, and both the data and the reason for failure were recorded. Caries in a non-filled surface of a tooth with an acceptable RBC restoration was not considered reason for failure. Patient variables recorded at baseline were age and gender, the consumption of coffee/tee/cola, smoking and bruxism. Individuals who before the examination reported smoking and consumption of any type of beverages with discoloring effect (coffee, tea, cola) at least once per day during the year were classified as current smokers and/or beverage consumers. The diagnosis of bruxism is based particularly on history, tooth mobility, tooth wear (attrition or chipping) and other clinical findings. For history taking the patients had to fill a questionnaire where questions were focusing on the night or awake grinding, jaw fatigue on awakening or on the experience temporal headache. The clinical examination covered the detection of tooth wear seen within the normal range of jaw movements or especially at eccentric position. Masseter muscle hypertrophy, masticatory muscle discomfort, tooth hypersensitivity to cold, tooth abfraction, clicking of the temporomandibular joint and tongue or cheek indentation. Also "possible" bruxers were considered to have bruxism. For the tooth examination the surface was dried with an air stream before evaluation, except for color scoring. Approximal surface control was performed with the help of a dental floss. In order to avoid unnecessary radiation exposure radiographs 


\begin{tabular}{lll}
\multicolumn{3}{l}{ Table 3 - Descriptive data of variables and failures. } \\
$\left.\begin{array}{lll}\text { Independent variables } & \mathrm{n}(\%) & \text { Failure - } \mathrm{n}(\%) \\
\hline \begin{array}{l}\text { Tooth type } \\
\text { Central incisor }\end{array} & 102(62.6) & 15(14.7) \\
\text { Lateral incisor } & 53(32.5) & 4(6.6) \\
\text { Canine } & 8(4.9) & 0(0) \\
\text { Total } & 163(100) & 19(11.7) \\
\text { Build-up type } & & \\
\text { Fracture } & 92(56.4) & 14(15.2) \\
\text { Diastema closure } & 71(43.6) & 5(7.0) \\
\text { Total } & 163(100) & 19(11.7) \\
\text { Build-up size } & & \\
<25 \% & 97(59.5) & 10(10.3) \\
\text { 25-50\% } & 48(29.4) & 7(14.6) \\
>50 \% & 18(11.1) & 2(11.1) \\
\text { Total } & 163(100) & 19(11.7) \\
\text { Composite type } & & \\
\text { Microhybrid } & 85(52.1) & 11(12.9) \\
\text { Nanofill } & 78(47.9) & 8(10.3) \\
\text { Total } & 163(100) & 19(11.7) \\
\text { Follow-up time } & & \\
8 \text {-10 years } & 35(21.5) & 3(8.6) \\
\text { 5-7 years } & 128(78.5) & 16(12.5) \\
\text { Total } & 163(100) & 19(11.7) \\
\hline & & \\
& & \\
& & \end{array}\right)$ \\
\hline
\end{tabular}

were only made in those cases when the clinical examination indicated so and it was necessary for the completion of the examination [17].

The data collection and the statistical analysis were performed using SPSS for Windows 23.0 (SPSS, Chicago, IL, USA). The frequency distributions of the evaluated criteria and the reasons for failure were described by descriptive statistics. Qualitative analysis based on the modified USPHS criteria was analyzed independently for each of the 12 evaluated clinical characteristics. Differences in the qualitative criteria between the materials were analyzed using Fisher's Exact Test. Because of the cluster-effect related to the multiple restoration in some individual and its contextual variables the average event rates were modeled and compared with a "shared frailty" model. This model is an extension of the Cox proportional hazard model that includes a frailty term to take the contextual dependency of events within into account. Hazard ratios (HR) with respective $95 \%$ confidence intervals (CI) were determined.

Survival analysis was performed using the Kaplan-Meier statistical method to obtain the survival curves for the variables of interest, followed by Log-Rank test for comparison between groups. $p$ values less than $5 \%$ were considered to be statistically significant in all applied tests.

\section{Results}

In the present study, a total of 163 anterior direct $\mathrm{RBC}$ restorations were evaluated in 65 adult patients. The follow-up time varied from 5 to 10 years with a mean observation time of $7.2( \pm 1.4)$ years. The distribution of restorations and failures according to the independent variables is shown in Table 3. Table 4 shows the summaries of USPHS scores according to the RBC type, restoration size and type of build-up. All scores

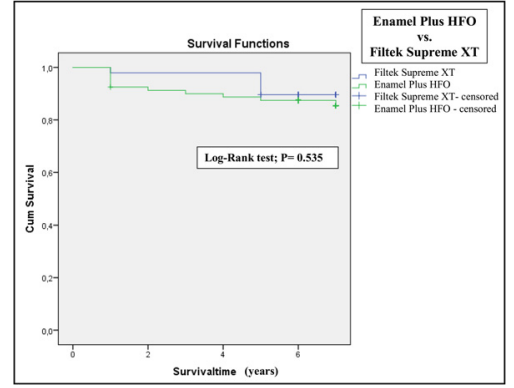

Fig. 1 - Kaplan-Meier survival curve for survival of nanofill and microhybrid direct $R B C$ restorations during the mean observation period.

for those restorations requiring repair or replacement, were considered clinically unacceptable and were considered as failures in analysis. When a restoration had failed before the examination, the date and reason for the failure was recorded from the patient's dental record. Of the 163 restorations, 19 (11.66\%) were determined to be unacceptable. The annual failure rate for the mean observation period is $1.43 \%(0.00-3.34 \%)$. The reasons for failure included fracture of restoration, color mismatch and marginal discoloration (Table 5). In two cases the restoration was considered to be failure, because two unacceptable changes were present simultaneously. Thus, the number of unacceptable defects is 21 , meanwhile the number of failed restorations is 19 .

The overall survival during the registration period was $88.34 \%$. The failure rates for Filtek Supreme XT and Enamel Plus HFO were $12.8 \%$ and $12.9 \%$, respectively, after 10 years. The Extended Cox analysis with shared frailty revealed that the overall difference in potential hazard of using Enamel Plus HFO or Filtek Supreme XT was not significant at $5 \%(p=0.704)$. Fig. 1 shows the Kaplan-Meier survival curve for the 2 materials over the mean observation period (7.2 years) of service. Nanofill restorations had significantly higher rate of color mismatch $(p=0.002)$, however microhybrids more frequently failed due to fracture of restoration $(p=0.034)$. The build-ups performed with Filtek Supreme XT nanofill RBC had a risk of failure because of color mismatch of 8.3 times (95\% CI: 1.8; 38.1) higher than those build-ups made from Enamel Plus HFO microhybrid RBC. On the other hand, the risk of failure due to chipping is 3.7 times higher (95\% CI: 0.9; 18.9) for Enamel Plus HFO compared to Filtek Supreme XT. The occurrence of chipping or fracture of restoration within one year after the placement was $57.1 \%$ ( $p=0.036$; 95\% CI: $2.1 ; 37.9)$.

A total of $103(63.2 \%)$ restorations were accepted without detectable changes and clinical remarks ("0" score), and in $60(36.8 \%)$ cases, at least 1 detectable change was found ("1, 2,3 " score). The most frequent failures were the color mismatch $(9.2 \%)$ and fracture of restoration (8.6\%). In $8.6 \%$ of the cases, slight or obvious staining of the restoration margin was observed and in $3.7 \%$ minor defects were found at the margins. The loss of surface gloss or slightly rough surface was found in $6.1 \%$. Only one tooth $(0.6 \%)$ showed sensitivity during the examination. For the category of "fracture of tooth", "wear of restoration or antagonist" and for "caries" all the 163 restorations were accepted without qualitative deterioration 
Table 4 - Summaries of USPHS criteria according to the composite type, restoration size and type.

\begin{tabular}{|c|c|c|c|c|c|c|c|}
\hline \multirow[b]{2}{*}{ USPHS criteria } & \multicolumn{2}{|c|}{ Type of material } & \multicolumn{2}{|c|}{ Type of build-up } & \multicolumn{3}{|c|}{ Size of restoration ${ }^{*}$} \\
\hline & $\begin{array}{l}\text { Nanofill } \\
\mathrm{n}=78\end{array}$ & $\begin{array}{l}\text { Microhibrid } \\
\mathrm{n}=85\end{array}$ & $\begin{array}{l}\text { Fracture } \\
\mathrm{n}=92\end{array}$ & $\begin{array}{l}\text { Diastema } \\
\mathrm{n}=71\end{array}$ & $\begin{array}{l}<25 \% \\
\mathrm{n}=97\end{array}$ & $\begin{array}{l}25-50 \% \\
n=48\end{array}$ & $\begin{array}{l}>50 \% \\
\mathrm{n}=18\end{array}$ \\
\hline \multicolumn{8}{|c|}{ Marginal adaptation } \\
\hline 0 & 75 & 82 & 86 & 71 & 93 & 46 & 18 \\
\hline 1 & 3 & 3 & 6 & 0 & 4 & 2 & 0 \\
\hline 2 & 0 & 0 & 0 & 0 & 0 & 0 & 0 \\
\hline 3 & 0 & 0 & 0 & 0 & 0 & 0 & 0 \\
\hline 4 & 0 & 0 & 0 & 0 & 0 & 0 & 0 \\
\hline \multicolumn{8}{|l|}{ Color match } \\
\hline 0 & 62 & 83 & 83 & 65 & 89 & 43 & 16 \\
\hline 1 & 7 & 2 & 5 & 4 & 5 & 2 & 2 \\
\hline 2 & 6 & 0 & 4 & 2 & 3 & 3 & 0 \\
\hline 3 & 0 & 0 & 0 & 0 & 0 & 0 & 0 \\
\hline 4 & 0 & 0 & 0 & 0 & 0 & 0 & 0 \\
\hline \multicolumn{8}{|c|}{ Marginal discoloration } \\
\hline 0 & 71 & 77 & 83 & 67 & 92 & 40 & 17 \\
\hline 1 & 6 & 8 & 8 & 4 & 5 & 7 & 1 \\
\hline 2 & 1 & 0 & 1 & 0 & 0 & 1 & 0 \\
\hline 3 & 0 & 0 & 0 & 0 & 0 & 0 & 0 \\
\hline \multicolumn{8}{|c|}{ Surface roughness } \\
\hline 0 & 76 & 77 & 88 & 65 & 91 & 46 & 16 \\
\hline 1 & 2 & 8 & 4 & 6 & 6 & 2 & 2 \\
\hline 2 & 0 & 0 & 0 & 0 & 0 & 0 & 0 \\
\hline 3 & 0 & 0 & 0 & 0 & 0 & 0 & 0 \\
\hline \multicolumn{8}{|c|}{ Fracture of restoration } \\
\hline 0 & 75 & 74 & 82 & 67 & 89 & 44 & 16 \\
\hline 1 & 2 & 5 & 7 & 0 & 7 & 0 & 0 \\
\hline 2 & 1 & 6 & 3 & 4 & 1 & 4 & 2 \\
\hline 3 & 0 & 0 & 0 & 0 & 0 & 0 & 0 \\
\hline \multicolumn{8}{|l|}{ Fracture of tooth } \\
\hline 0 & 78 & 85 & 92 & 71 & 97 & 48 & 18 \\
\hline 1 & 0 & 0 & 0 & 0 & 0 & 0 & 0 \\
\hline 2 & 0 & 0 & 0 & 0 & 0 & 0 & 0 \\
\hline 3 & 0 & 0 & 0 & 0 & 0 & 0 & 0 \\
\hline \multicolumn{8}{|c|}{ Wear of restoration } \\
\hline 0 & 78 & 85 & 92 & 71 & 97 & 48 & 18 \\
\hline 1 & 0 & 0 & 0 & 0 & 0 & 0 & 0 \\
\hline \multicolumn{8}{|c|}{ Wear of antagonist } \\
\hline 0 & 78 & 85 & 92 & 71 & 97 & 48 & 18 \\
\hline 1 & 0 & 0 & 0 & 0 & 0 & 0 & 0 \\
\hline \multicolumn{8}{|l|}{ Caries } \\
\hline 0 & 78 & 85 & 92 & 71 & 97 & 48 & 18 \\
\hline 1 & 0 & 0 & 0 & 0 & 0 & 0 & 0 \\
\hline \multicolumn{8}{|c|}{ Postop. sensitivity } \\
\hline 0 & 78 & 84 & 91 & 71 & 97 & 47 & 18 \\
\hline 1 & 0 & 1 & 1 & 0 & 0 & 1 & 0 \\
\hline 2 & 0 & 0 & 0 & 0 & 0 & 0 & 0 \\
\hline 3 & 0 & 0 & 0 & 0 & 0 & 0 & 0 \\
\hline
\end{tabular}

*Extension of the restoration related to the entire clinical crown surface.

Gray band shows the clinically not acceptable scores

\section{Table 5 - Failed restorations by material and type of build-up during the 10-year monitoring period.}

\begin{tabular}{|c|c|c|c|c|c|c|c|c|c|c|c|c|c|c|c|}
\hline \multirow[t]{2}{*}{ Cause of failure } & \multicolumn{2}{|c|}{ Materials } & \multicolumn{2}{|c|}{ Type of build-up } & \multicolumn{11}{|c|}{ Time of failure (years) } \\
\hline & Microhybrid & Nanofill & Fracture & Diastema & 1 & 2 & 3 & 4 & 5 & 6 & 7 & 8 & 9 & 10 & Total \\
\hline Restoration fracture & 11 & 3 & 10 & 4 & 8 & 1 & 1 & 1 & 1 & 0 & 1 & 0 & 0 & 1 & 14 \\
\hline Color mismatch & 0 & 6 & 4 & 2 & 0 & 0 & 0 & 0 & 4 & 0 & 2 & 0 & 0 & 0 & 6 \\
\hline Marginal discoloration & 0 & 1 & 1 & 0 & 0 & 0 & 0 & 0 & 1 & 0 & 0 & 0 & 0 & 0 & 1 \\
\hline Total & 11 & 10 & 15 & 6 & 8 & 1 & 1 & 1 & 6 & 0 & 3 & 0 & 0 & 1 & $21 / 19^{a}$ \\
\hline
\end{tabular}

${ }^{a}$ One nanofill fracture build-up failed because of fracture and color mismatch at the same time and one nanofill diastema closure failed because of color mismatch and marginal discoloration at the same time. The number of unacceptable changes is 21 , however the number of failed restorations is 19 . 
(score "0"). None of the evaluated teeth needed endodontic treatment during the examination period.

The incidence of the unfavorable events was similar for the investigated materials, $32.1 \%$ when the restorations were made with Filtek Supreme XT and 32.9\% with Enamel Plus HFO. Considering the type or size of the restoration, the incidence of the qualitative deteriorations or failures was not significantly different, neither in case of fracture build-ups compared to diastema closure $(p=0.437 ; 95 \%$ CI: $0.09 ; 2.75)$, nor in case of small $(<25 \%)$, medium $(25-50 \%)$ or large $(>50 \%)$ restorations ( $p=0.395 ; 95 \% \mathrm{CI}: 0.10 ; 2.47)$. No correlation was found between the type of tooth (central vs. lateral incisor vs. canine) and type of restoration (fracture build-up vs. diastema closure) with respect to the failures ( $p=0.362 ; 95 \% \mathrm{CI}$ : 0.50 ; 0.53). Furthermore, dietary habits, like tea/coffee/cola consumption was observed to lead to a statistically significant more frequent occurrence of color mismatch $(p=0.005 ; 95 \%$ CI: $0.10 ; 0.67)$. On the other hand, smoking $(p=0.732)$ or bruxism ( $p=0.054)$ were not observed as an influencing factor on the appearance of detectable changes (Table 6).

\section{Discussion}

In this retrospective clinical study, the long-term clinical performance of a nanofill and a microhybrid RBC restorative material applied in anterior restorations was analyzed over an extended period of time. Furthermore, the effect of the type of direct $\mathrm{RBC}$ restoration (fracture or diastema), the size of restoration $(<25 \%, 25-50 \%,>50 \%)$, the presence of bruxism, coffee/tea/cola consumption and smoking on the restoration's longevity was investigated. A satisfying clinical performance was observed for direct RBC restorations, with an annual failure rate of $1.43 \%$ after a mean observation period of 7.2 years. However, results and survival analysis regarding differences between materials and groups should be interpreted with care as the case number and number of failures are limited.

As Demarco et al. referred in their systematic review, in spite of the frequent use of RBC in the anterior area, there is a lack of long term results from clinical trials regarding the performance of anterior restorations [8]. The reasons of the low number of long-term studies that are time consuming and factors such as patient compliance, recall failure make these investigations complicated [18]. In the present study the recall rate was $79 \%$ up to 10 years (5-10 years).

In contrast with a prospective, randomized clinical trial, a retrospective designed study may result in some deficiencies, such as the incomplete standardization of indications, treatment protocols or the missing baseline scoring. However, in the present study the involved practitioner was a university instructor, specialized in esthetic dentistry, working according to high standards. Moreover, the selected patient group in this study also acted as presentational cases for demonstrations for undergraduate and graduate education, therefore requiring an excellent implementation.

Regarding the method used to evaluate the restorations, the modified USPHS system was applied, which enables a standardized and detailed evaluation of restorations overtime. Although, this method has some limitations, it is often used in clinical studies and allows comparisons among them.
Considering the overall service time, this study reported an $88.34 \%$ survival rate of anterior RBC restorations which is similar to the studies with 4-7 years' follow-up time. For instance, Al-Khayatt et al. [19] showed $85 \%$, Frese et al. [1] $84.6 \%$ and Gresnigt et al. [9] found $87.5 \%$ of survival in their longitudinal clinical study. The most common reasons for failure in this study was the color mismatch and the cohesive chipping of the RBC. The previously mentioned studies similarly concluded, that the anterior restorations failed more due to esthetic reasons or fracture as well $[1,3,9]$. However, in our study the evaluation was more stringent than in the other similar studies, considering the more prominent nature of the anterior teeth where any little unfavorable event can disturb the esthetic appearance. From the USPHS code 2 for each category and even code 1 for fracture were considered as failure, meanwhile in other studies codes 3-4 or 4-5 were considered as failure. However, it is important to note that not in all cases of failures were composite build-up entirely replaced, often the life of the restoration was extended with a simple repair, thanks to the resin composite's material properties. Frese et al. [1] defined the repaired cases as survival not as failure or success. According to their $100 \%$ functional survival rate, it was concluded that the reparability of the RBC materials is the most important factor in prolonging the life of the restorations, providing a patient/tooth-oriented treatment approach in anterior direct $\mathrm{RBC}$ restorations [1]. Our results showed, that significantly more incisal cohesive chipping occurred within the $\mathrm{RBC}$ in case of microhybrid RBC build-up. It is 3.7 times higher risk of failure for microhybrid Enamel Plus HFO than the restorations made with nanofill Fitek Supreme XT. Frese et al. [1], van Dijken et al. [11], Coelho-de-Souza et al. [20], Gresnigt et al. [9] and Milosevic and Burnside [21] also reported, that fracture or chipping is the most frequent reason for failure in microhybrid anterior RBC restorations. In comparison with nanofill RBCs the microhybrid's structure is less homogen, caused by the different size of filler particles, thus cohesive fracture may occur in higher rate within the material. In our investigation the date of chipping was also documented and it was found that in $57.1 \%$ the fracture occurred within one year after the placement of restoration. During everyday mastication the overloaded occlusal contacts of the restoration chipped within a short time of wear, however, after repair these contacts were carefully removed to avoid further chipping. One of the examined factors which may influence the fracture of tooth or restoration, thus the longevity of the build-up, was bruxism and no correlation was determined. In accordance with our results, Milosevic and Burnside [21] also found that the bruxism itself was not related to failure, such as fracture of tooth or RBC. In contrast, van Dijken et al. reported a significantly higher rate of fracture in bruxing patients compared to those who are not suffering from bruxism [11]. Although, bruxism is a self-reported factor which may not be accurately detected by patients and the diagnosis of etiological factors from patient histories can be difficult and imprecise.

Besides the restoration fracture, color mismatch was the other major cause of failure in this study. The risk of failure due to color mismatch was 8.3 times higher when the restoration was performed with nanofill Filtek Supreme XT. Yazici et al. and Nasim et al. [22,23] compared the color stability of micro- 
Table 6 - Extended Cox regression analysis with adjusted Hazard Ratio (HR) for independent variables and failures of direct RBC restorations.

\begin{tabular}{llll}
\hline Independent variables & HR & $95 \%$ CI & $p$-value \\
\hline Sex (female vs. male) & 1.27 & $0.35-4.54$ \\
Tooth type (central incisor vs. lateral incisor/canine) & 0.48 & $0.15-1.55$ & 0.718 \\
Type of build-up (fracture vs. diastema) & 1.94 & $0.09-2.75$ & 0.219 \\
Size of build-up (<50\% vs. >50\%) & 0.50 & $0.10-2.47$ & 0.437 \\
Material (nanofill vs. microhybrid) & 0.83 & $0.31-2.22$ & 0.395 \\
Time of follow-up (5-7 vs. 8-10 years) & 0.42 & $0.09-2.03$ & 0.704 \\
Beverages (yes vs. no) & 3.87 & $0.10-0.67$ & 0.278 \\
Smoking (yes vs. no) & 0.72 & $0.11-4.83$ & 0.005 \\
Bruxism (yes vs. no) & 3.18 & $0.98-10.29$ & 0.732 \\
\hline Abbreviations; RBC, resin based composite; 95\%CI, 95\% confidence interval; clustered variable is the individual.
\end{tabular}

hybrid, microfill and nanofill RBCs in an in vitro investigation and found that microhybrid seemed to be the most stable type, meanwhile nanofills showed the highest rate of discoloration in tea after a period of 7 and 30 days. Tekçe et al. had similar findings in their in vitro study with higher susceptibility of nanofills to color alteration in beverages [24]. In the oral environment, superficial degradation of the restorative materials and their absorption of staining agents can cause discoloration [25]. Supposedly, the color change can easily be related to the nature of the resin matrix. The presence of low TEGDMA content may limit water uptake and, consequently, color variations induced by the absorption of the staining solution [26]. However, in this study both the compared RBCs contain TEGDMA, thus TEGDMA content could not be the main explanation of staining. Likewise, the filler content seems to play an important role in RBC color stability [27]. A previous study showed that the filler particle size and distribution seem to be directly correlated to optical properties and that nanofiller particles provide low visual opacity in non-pigmented dental RBCs [28]. Moreover, a smaller filler size might contribute to decrease staining and enhance esthetic appearance [26]. However, according to our long-term in vivo results, nanoparticles did not show beneficial properties to the color stability and surface luster. This is corroborated by a recent systematic review showing that the most recently introduced nanofill RBCs have not shown superiority in color stability and retention of gloss compared to microhybrids [29]. Nanofill RBCs have higher volumetric filler content (55\%) with nano-sized filler particles and nanoclusters, thus the amount of coupling agent between the matrix and the fillers and inside the nanoclusters is higher in nanofill resin composites. The hydrolysis of the silane coupling agent is widely accepted as one of the main causes of RBC material degradation [30,31], leading to a shortened service life of dental restorations. Consequently, in nanofills the rate of degradation and susceptibility to color alteration may be more prominent, leading to clinically perceptible color change. Moreover, significantly higher rate of color alteration was observed in those patients who referred daily coffee/tee consumption. As the aforementioned in vitro studies have shown the color of composite resin was not stable when exposed to various staining media, especially it was true for nanofill RBCs.

Besides failures, marginal discoloration and surface roughness were the common clinically acceptable changes observed. From the fourteen marginal discoloration (8.4\%) only one case was unaccepted clinically. Marginal discoloration and detectable margins are the only clinically measurable signs for the evaluation of the marginal seal of direct RBC restorations. Margins of fracture build-ups and diastema closures are located in enamel, thus the bonding to enamel is crucial for good seal and for this purpose the enamel etching with $37 \%$ phosphoric acid is the best method to establish a micro-retentive pattern, as it was concluded in a meta-analysis of direct anterior restorations by Heintze et al. [32]. Nowadays, it is often recommended to prepare a long bevel, especially on the labial surface, to make transition between restoration and enamel almost invisible. In our study, the teeth were beveled prior to conditioning the enamel, and the broad bevel may also reduce the number of restorations with marginal staining. The other benefit of the long bevel may be the higher fracture resistance of the RBC restoration at the tooth-restoration interface as it was proved in a laboratory study [33]. It was also found in our clinical trial that none of the restorations had failed due to bulk fracture of the restoration or due to loss of retention.

Loss of surface gloss or the appearance of slight roughness was observed in $6.1 \%$. These deteriorations were clinically acceptable and easily remediable with polishing. Furthermore, our results did not find statistically significant differences in surface roughness between the two examined materials.

Deterioration of marginal integrity was found in a few cases (3.7\%) which were independent of the type of the material. The clinically acceptable change was the detectability of the restoration's margin with a probe. The reason of this marginal imperfection could be the abrasion of the very thin restorative material from the bevel margin.

Together with fracture secondary caries is considered a major reason for failure in posterior RBC restorations [4,34]. Results of the present study indicate that secondary caries is not a major cause for failure of anterior restorations, actually, there were no secondary caries detected over the examination period.

In accordance with Gresnigt et al. [9] neither clinically detectable wear of restoration nor wear of antagonist was observed in our study. Maybe due to the small filler particle components of the investigated materials. However, it should be mentioned that the criteria were used to evaluate wear are not detailed, though, diagnosis of wear could be difficult, considering the multifactorial etiology of this phenomenon. 
Postoperative sensitivity was only seen in one case out of the total 163 teeth. The size of the reparable broken pieces, thus the size of the exposed dentin surface was not an influencing factor in postoperative sensitivity. Moreover, the total-etch technique was used for the adhesion of each restoration. The etching of dentin with phosphoric acid is considered as a crucial factor in postoperative sensitivity [35], however our results cannot confirm this statement. Endodontic complication did not occur in any of the teeth.

Despite the excellent properties of nanofill RBCs, their clinical performance and survival rate was not superior to that of the microhybrid RBCs. Considering the type and size of the build-ups it was found that these variables did not affect the clinical outcome of the restorations. However, as a limitation, the distribution of small, medium and large restorations was not equal and the good results may be influenced by the higher number of smaller build-ups. Further evaluation with increased number of large restorations is necessary to overcome this limitation.

\section{Conclusions}

Within the limitations of this retrospective study, the following conclusions can be drawn:

1) The microhybrid and nanofill RBCs showed acceptable clinical durability in anterior restorations with $1.43 \%$ AFR for the 7.2 years mean observation time.

2) The most frequent failure was RBC fracture and color mismatch of the restoration. Fracture of the restorations occurred 3.7 times more often with microhybrid restorations, meanwhile color mismatch was 8.3 times more frequent with nanofill RBCs.

3) Coffee/cola/tee consumption had direct impact on nanofill restoration's color mismatch.

4) Size and type of the restoration, bruxism and smoking were found as factors not affecting the deterioration of the restorations.

\section{Acknowledgments}

This work was supported by Pécsi Tudományegyetem Általános Orvostudományi Kar Kutatási-Alap-2016/1 Research Grant. The present scientific contribution is dedicated to the 650th anniversary of the foundation of the University of Pécs, Hungary.

\section{REFERENCES}

[1] Frese C, Schiller P, Staehle HJ, Wolff D. Recontouring teeth and closing diastemas with direct composite buildups: a 5-year follow-up. J Dent 2013;11:979-85.

[2] Ferracane JL. Resin composite—state of the art. Dent Mater 2011;27:29-38.

[3] Baldissera RA, Corrêa MB, Schuch HS, Collares K, Nascimento GG, Jardim PS, et al. Are there universal restorative composites for anterior and posterior teeth? J Dent 2013;41:1027-35.
[4] Lempel E, Tóth Á, Fábián T, Krajczár K, Szalma J. Retrospective evaluation of posterior direct composite restorations: 10 years findings. Dent Mater 2015;31: 115-22.

[5] Wolff D, Kraus T, Schach C, Pritsch M, Mente J, Staehle HJ, et al. Recontouring teeth and closing diastemas with direct composite buildups: a clinical evaluation of survival and quality parameters. J Dent 2010;38:1001-9.

[6] Da Rosa Rodolpho PA, Donassollo TA, Cenci MS, Loguércio AD, Moraes RR, Bronkhorst EM, et al. 22-year clinical evaluation of the performance of two posterior composites with different filler characteristics. Dent Mater 2011;27:955-63.

[7] Pallesen U, van Dijken JWV, Halken J, Hallosten AL, Höigaard R. Longevity of posterior resin composite restorations in permanent teeth in Public Dental Health Service: a prospective 8 years follow up. J Dent 2013;41:297-306.

[8] Demarco FF, Collares K, Coelho-de-Souza FH, Correa MB, Cenci MS, Moraes RR, et al. Anterior composite restorations: a systematic review on long-term survival and reasons for failure. Dent Mater 2015;31:1214-24.

[9] Gresnigt MM, Kalk W, Ozcan M. Randomized controlled split-mouth clinical trial of direct laminate veneers with two micro-hybrid resin composites. J Dent 2012;9:766-75.

[10] Alonso V, Caserio M. A clinical study of direct composite full-coverage crowns: long-term results. Oper Dent 2012;37:432-41.

[11] van Dijken JWV, Pallesen U. Fracture frequency and longevity of fractured resin composite, polyacid-modified resin composite, and resin modified glass ionomer cement Class IV restorations: an up to 14 years of follow-up. Clin Oral Investig 2010;14:217-22.

[12] Peumans M, Van Meerbeek B, Lambrechts P, Vanherle G. The 5-year clinical performance of direct composite additions to correct tooth form and position. II. Marginal qualities. Clin Oral Investig 1997;1:19-26.

[13] Kubo S, Kawasaki A, Hayashi Y. Factors associated with the longevity of resin composite restorations. Dent Mater J 2011;30:374-83.

[14] Opdam NJM, Bronkhorst EM, Loomans BA, Hujsmans MC. 12-year survival of composite vs. amalgam restorations. J Dent Res 2010;89:1063-7.

[15] Da Rosa Rodolpho PA, Cenci MS, Donassollo TA, Loguercio AD, Demarco FF. A clinical evaluation of posterior composite restorations: 17-year findings. J Dent 2006;34:427-35.

[16] Vanini L, Mangano FM. Determination and communication of color using the five color dimensions of teeth. Pract Proced Aesthet Dent 2001;13:19-26.

[17] European Commission. European guidelines on radiation protection in dental radiology. The safe use of radiographs in dental practice. Radiat Prot 2004:136.

[18] Manhart J, Chen H, Hamm G, Hickel R. Buonocore memorial lecture. Review of the clinical survival of direct and indirect restorations in posterior teeth of the permanent dentition. Oper Dent 2004;29:481-508.

[19] Al-Khayatt AS, Ray-Chaudhuri A, Poyser NJ, Briggs PF, Porter RW, Kelleher MG, et al. Direct composite restorations for the worn mandibular anterior dentition: a 7-year follow-up of a prospective randomized controlled split-mouth clinical trial. J Oral Rehabil 2013;40:389-401.

[20] Coelho-de-Souza FH, Gonçalves DS, Sales MP, Erhardt MCG, Corrêa MB, Opdam NJM, et al. Direct anterior composite veneers in vital and non-vital teeth: a retrospective clinical evaluation. J Dent 2015;11:1330-6.

[21] Milosevic A, Burnside G. The survival of direct composite restorations in the management of severe tooth wear including attrition and erosion: a prospective 8-year study. J Dent 2015;14:13-9. 
[22] Yazici AR, Celik C, Dayangaç B, Ozgünaltay G. The effect of curing units and staining solutions on the color stability of resin composites. Oper Dent 2007;32:616-22.

[23] Nasim I, Neelakantan P, Sujeer R, Subbarao CV. Color stability of microfilled, microhybrid and nanocomposite resins-an in vitro study. J Dent 2010;38:137-42.

[24] Tekçe N, Tuncer S, Demirci M, Serim ME, Baydemir C. The effect of different drinks on the color stability of different restorative materials after one month. Restor Dent Endod 2015;40:255-61.

[25] Bagheri R, Burrow MF, Tyas M. Influence of food-simulating solutions and surface finish on susceptibility to staining of aesthetic restorative materials. J Dent 2005;33:389-98.

[26] Vichi A, Ferrari M, Davidson CL. Color and opacity variations in three different resin-based composite products after water aging. Dent Mater 2004;20:530-4.

[27] Rodrigues Jr SA, Scherrer SS, Ferracane JL, Della Bona A. Microstructural characterization and fracture behavior of a microhybrid and a nanofill composite. Dent Mater 2008;24:1281-8.

[28] Mitra SB, Wu D, Holmes BN. An application of nanotechnology in advanced dental materials. J Am Dent Assoc 2003;134:1382-90.
[29] Kaizer MR, de Oliveira-Ogliari A, Cenci MS, Opdam NJ, Moraes RR. Do nanofill or submicron composites show improved smoothness and gloss? A systematic review of in vitro studies. Dent Mater 2014;30:e41-78.

[30] Ferracane JL, Marker VA. Solvent degradation and reduced fracture toughness in aged composites. J Dent Res 1992;71:13-9.

[31] Söderholm KJ, Zigan M, Ragan M, Fischlschweiger W, Bergman M. Hydrolytic degradation of dental composites. J Dent Res 1984;63:1248-54.

[32] Heintze SD, Rousson V, Hickel R. Clinical effectiveness of direct anterior restorations-a meta-analysis. Dent Mater 2015;31:481-95.

[33] Tan DE, Tjan AH. Margin designs and fracture resistance of incisal resin composite restorations. Am J Dent 1992;5:15-8.

[34] Demarco FF, Correa MB, Cenci MS, Moraes RR, Opdam NJM. Longevity of posterior composite restorations: not only a matter of materials. Dent Mater 2012;28:87-101.

[35] Gordon JC. Preventing postoperative tooth sensitivity in Class I, II and V restorations. JADA 2002;133:229-31. 